\title{
MACROPROYECTOS DE INTERÉS SOCIAL NACIONAL EN CIUDADES INTERMEDIAS Mecanismo para reconfigurar y desarrollar el tejido social y la estructura urbana
}

\author{
Autora: Luz Adriana Castiblanco Martínez \\ Universidad Nacional de Colombia \\ Email: lacastiblancom@gmail.com, luz.castiblanco622@esap.gov.co \\ Co-autor: Jeison Andrés Hincapie Rodriguez \\ Universidad Nacional de Colombia \\ Email: jeahincapiero@unal.edu.co, jeian91@gmail.com
}

\section{RESUMEN}

Los Macroproyectos de Interés Social Nacional (MISN) surgen como alternativas para mitigar el déficit habitacional en las ciudades, dado que incorporan instrumentos de planeación, financiación y gestión del suelo para materializar una operación de gran escala que permita el desarrollo territorial en diferentes zonas del territorio colombiano; sin embargo su implementación, especialmente en ciudades intermedias como Manizales y Soacha, ha desencadenado cambios en las lógicas socio espaciales, dado que, de manera paralela, estas ciudades adquirieron la connotación de Ciudades Intermedias, su localización dentro de los flujos de capital propio de la región andina las convierte en estratégicas para el sistema de movilidad, pero adicionalmente porque fueron antecesoras de programas de mejoramiento barrial, los cuales no alcanzaron indicadores representativos de eficiencia y eficacia hacia la mejora de las condiciones de habitabilidad y calidad de vida de la población allí asentada.

Palabras clave: Macroproyectos, estructura urbana, sistemas estructurantes, planificación

\begin{abstract}
Macro-Projects of National Social Interest (MISN) appear as alternatives to mitigate the housing deficit in the cities, since they incorporate instruments of planning, financing and land management to materialize a large-scale operation that allows territorial development in different areas of the territory Colombian; however, their implementation, especially in intermediate cities such as Manizales and Soacha, has triggered changes in the spatial socio-logical ones, since in parallel these cities acquired the connotation of Intermediate Cities, their location within the flows of own capital of the Andean region Making them strategic for the mobility system, but also because they were the predecessors of neighborhood improvement programs, which did not reach indicators of efficiency and effectiveness towards improving the living conditions and quality of life of the population living there.
\end{abstract}

Key words: Macroprojects, urban structure, structuring systems, planning 


\section{INTRODUCCIÓN}

La comprensión de los procesos de transformación de las ciudades, partiendo de las ideas y teorías que dan cuenta del fenómeno urbano, hasta las estrategias de planeación y gestión que conllevan a la transformación del territorio, permite analizar y reflexionar sobre los proyectos urbanos, especialmente de los Macroproyectos de Interés Social Nacional (MISN), dada su estrecha relación con las políticas de vivienda emanadas desde el Ministerio de Vivienda, Ciudad y Territorio colombiano, pero sobre todo por la transformación que genera en las ciudades. Este tipo de intervenciones se orientan a la producción de vivienda de interés social (VIS) y vivienda de interés prioritario (VIP), cuyo mercado potencial se enfoca hacia población con bajo poder adquisitivo y/o en algunos casos víctimas del conflicto interno y de manera simultánea a disminuir el déficit habitacional de las ciudades en donde se implementan.

Considerando lo anterior, se tomaron como casos de estudio los Macroproyectos de Interés Social Nacional, en adelante MISN, San José en la ciudad de Manizales (Caldas) y Ciudad Verde en la ciudad de Soacha (Cundinamarca), especialmente por los cambios desencadenados en la forma de ocupación del territorio y su articulación con la ciudad, lo que ha generado afectaciones en el tejido social y la estructura urbana, De este modo, se pretende mostrar por un lado los impactos socioespaciales que genera la implementación y desarrollo de un MISN, y por el otro, cómo éstos inciden en tópicos como la sostenibilidad ambiental, las relaciones funcionales con la ciudad y la conformación de nuevos focos de desarrollo, generando disparidades entre la concepción del Macroproyecto y el modelo de ocupación territorial que había definido cada ciudad en su Plan de Ordenamiento Territorial; lo que a la postre transforma las lógicas de sin que necesariamente se cuente con una visión prospectiva del desarrollo.

En este sentido, si bien los MISN para su propuesta, diseño y ejecución, cuentan con un soporte técnico basado en estudios socioeconómicos, físicos y ambientales entre otros, que garantizan su realización; al revisar algunas de las etapas del proyecto se encuentran falencias en aspectos como la financiación, tiempos de ejecución, propuesta de diseño urbano y la provisión de equipamientos principalmente, lo que impide la consolidación de esta nueva pieza urbana con la estructura urbana de las ciudades que los albergan, pero sobre todo con las lógicas funcionales que han caracterizado el territorio y el tejido social que allí se ha conformado.

Partiendo de un análisis multitemporal, multidimensional y multiescalar, este documento pretende mostrar cómo la implementación de los MISN, que sin estar totalmente terminados luego de casi una década de ser adoptados, se han convertido en un mecanismo que ha acelerado procesos de gentrificación, migración y segregación socio espacial al interior de las ciudades objeto de estudio, en razón a que rompe con las lógicas propias del territorio y sus habitantes, pero también porque no consolida sistemas estructurantes como la estructura ecológica principal, movilidad y espacio público, de manera intraproyecto e intraciudad.

Finalmente y de manera complementaria, se señalaran aspectos propios del desarrollo de los MISN asociados con las etapas de ejecución, los Programas de Mejoramiento Integral que antecedieron la implementación de los MISN, la intencionalidad con que surgen, la idea de ciudad en la que se enmarcaron y el balance de los resultados obtenidos; para evidenciar su incidencia en las escalas local y regional, en contextos políticos, económicos, de gestión y su relación con agentes y preexistencias, lo que contribuirá a dar cuenta del cambio suscitado entre la idea de proyecto o intencionalidad y la transformación estructural de la ciudad o realización del mismo.

\section{CARACTERIZACIÓN MULTIDIMENSIONAL DEL MUNICIPIO DE SOACHA (CUNDINAMARCA)}

En un contexto histórico, los primeros planos elaborados en el año de 1572 sobre la distribución territorial de la Sabana, el río Bogotá fue tomado como eje central y a lo largo de él se estructuró el territorio. Soacha se estableció sobre el margen occidental del río, y por su ubicación geográfica, "parte baja de la Cuenca Alta del río Bogotá, fue el sitio indicado para la navegación y existió comunicación a través de una serie de pantanos y humedales similar al trazado de las vías actuales. A partir de Soacha se construyeron caminos que comunicaban con el Sumapaz y el Tequendama, hacia el oriente. Soacha fue centro de comercio y la entrada sur a la Sabana de Bogotá."(Lozada, 2000:1).

Hacia 1875 cambia su nombre a Soacha, se realiza el levantamiento del plano de la población, y es reconocido como Municipio del departamento de Cundinamarca. La construcción de la línea sur del Ferrocarril que conectaría 
el municipio con Bogotá se da hacia 1895, y el primer tren arribaría en 1898, constituyéndose en la tercera línea que se construía para la Capital, mejorando la comunicación, la cual hasta entonces se daba por medio de carrozas y movilización a caballo.

Desde el punto de vista físico, se encuentra ubicado en el área central del territorio nacional, sobre la cordillera oriental con una altitud que varía entre los 2.200 y los $3.800 \mathrm{msnm}$, hace parte de la provincia de Soacha junto con el municipio de Sibaté, y de la cuenca del río Bogotá y la subcuenca del río Soacha. Limita al Norte con los municipios de Bojacá y Mosquera, por el sur con Sibaté y Pasca, al Occidente con Granada y San Antonio del Tequendama y al Oriente Con Bogotá.

Tiene una superficie de $184,45 \mathrm{Km}^{2}$ cuadrados, de los cuales $19 \mathrm{Km}^{2}$ corresponden a la zona urbana dividida en 6 comunas y 328 barrios, y $165,45 \mathrm{Km}^{2}$ a la zona rural dividida en 2 corregimientos y 14 veredas; se divide en cuatro zonas geográficas que son: La cuenca alta del río de Soacha a partir del perímetro urbano, la zona urbana contenida al interior del perímetro urbano en la que se encuentran las 6 comunas, divididas en 348 barrios y la mayor zona de expansión del territorio, la cuenca del río Bogotá y la cuenca occidental del río Bogotá.
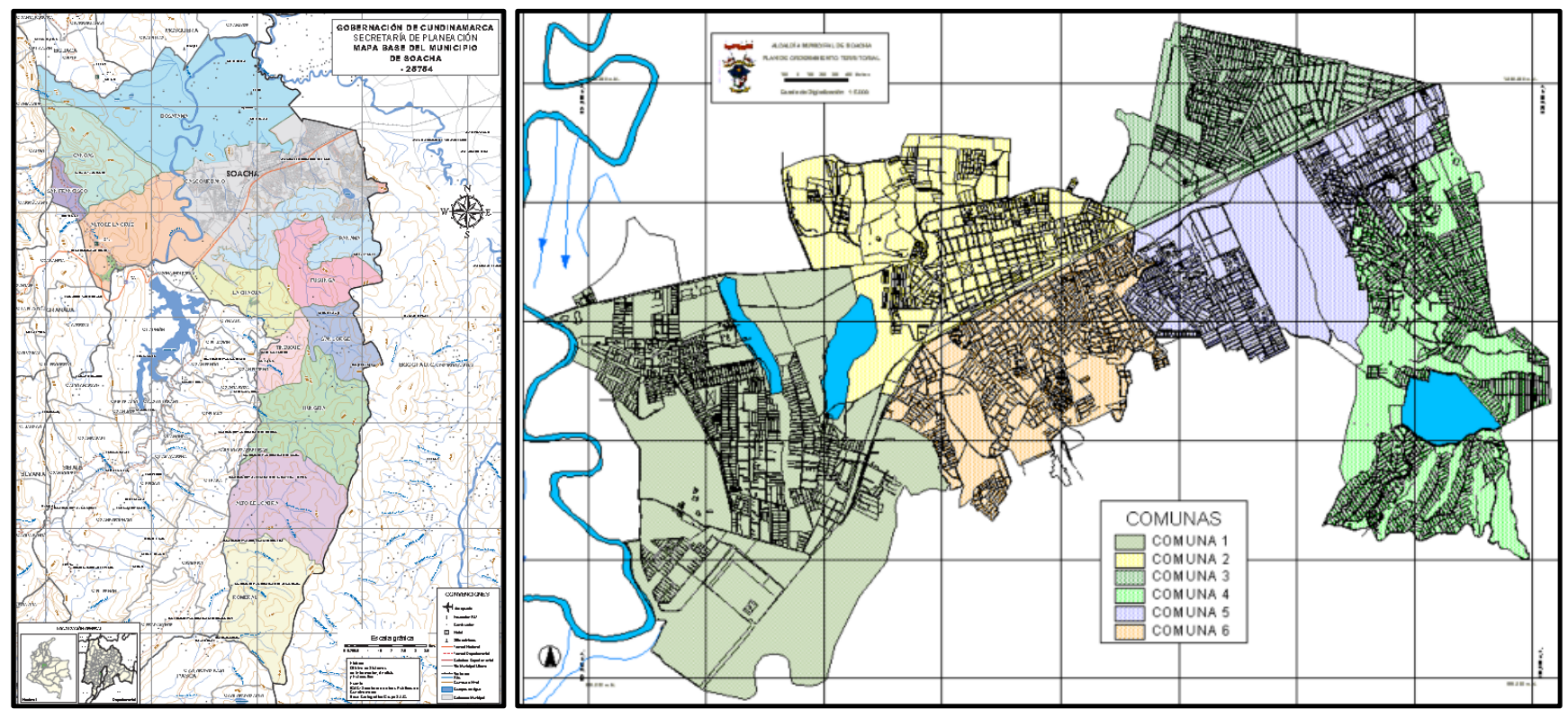

Mapa Base y Mapa de Comunas del Municipio de Soacha

Fuente: Tomado de http://www.cundinamarca.gov.co y https://www.emaze.com/@AILITCRQ/comuna-2-Soacha, 2016

De acuerdo con el Censo de 2005 el municipio contaba con 398.295 habitantes y para 2015 la población proyectada era 511.262 habitantes, lo que muestra un incremento aproximado del $29 \%$ en una década, de los cuales el $98,85 \%$ se ubica en el área urbana; sin embargo miembros de la comunidad aducen que la población del municipio estaría por encima del millón de habitantes, muchos en condición de vulnerabilidad social.

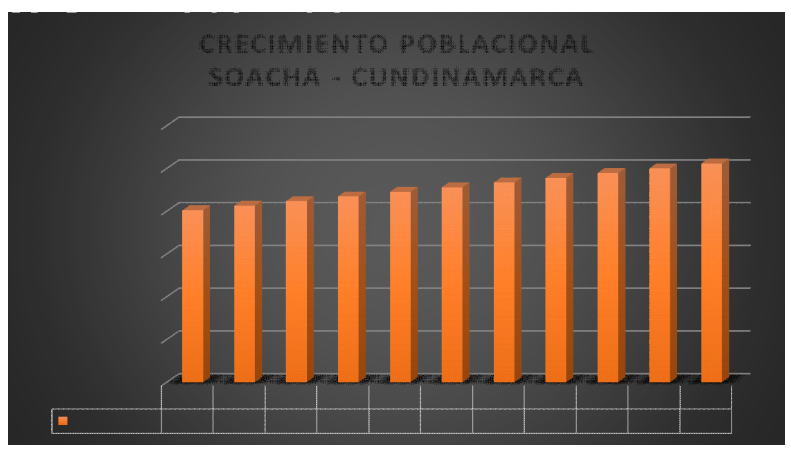

Crecimiento Poblacional

Elaboración propia a partir de datos DANE

El municipios maneja bajos índices de población afrocolombiana e indígena y cuenta con una densidad 
poblacional de 2.734,02 habitantes por $\mathrm{km}^{2}$, viabilizado principalmente por la características físicas de su territorio y el modelo de ocupación territorial utilizado desde su fundación, lo que ha contribuido a la conformación tanto de asentamientos formales e informales, los últimos como consecuencia de procesos migratorios durante los períodos de conflicto interno del país.

Debido a factores de orden público, entre 2006 y 2010 se desplazaron del municipio 945 personas, sin embargo en el mismo periodo recibió 13.407 personas que equivalen a 3.916 hogares, lo que se incide directamente sobre su crecimiento poblacional, dado que gran parte a los índices de migración, responden al conflicto interno, el valor del suelo como factor determinante dentro del mercado inmobiliario, procesos de parcelación y loteo ilegal (tierreros o invasiones), el crecimiento industrial y la proximidad con Bogotá lo que ha permitido que se configure una conurbación entre las dos entidades territoriales. Por tal motivo, su altísima densidad poblacional viene ejerciendo fuerte presión, no solo sobre el medio natural propiciando una degradación progresiva a los sistemas naturales, sino también sobre su estructura organizacional y sistema de equipamientos, lo que genera una carga en la provisión de bienes y servicios hacia ciertas localidades de Bogotá.

De acuerdo con el POT (Acuerdo No. 46 de 2000 "Por el cual se adopta el plan de ordenamiento territorial del municipio de Soacha), en el artículo 181 se establece que el Subsistema de Relación Regional está conformado por vías que conectan a los municipios aledaños con la Autopista sur, Avenida Indumil, Carretera a Sibaté, carretera a Mesitas del Colegio y la conexión con Bogotá. Y en el artículo 176 el tejido vial urbano está conformado por la Calle 13 (Avenida Luis Carlos Galán), la Avenida Suacha (Recorrido Rio) Carrera 13, Eugenio Díaz (Carrera 9 Este), San Mateo (Calle 30), Avenida Compartir (Carrera 19), Carrera 7 Centro, Avenida La Chucua, Avenida Ducales, Avenida Puerta Del Sol y Avenida Del Estadio. Y en el artículo 179 establece como subsistema de vial local la Avenida Ciudad de Cali, la Avenida circunvalar sur, la Avenida Variante Longitudinal y la Avenida Terreros.

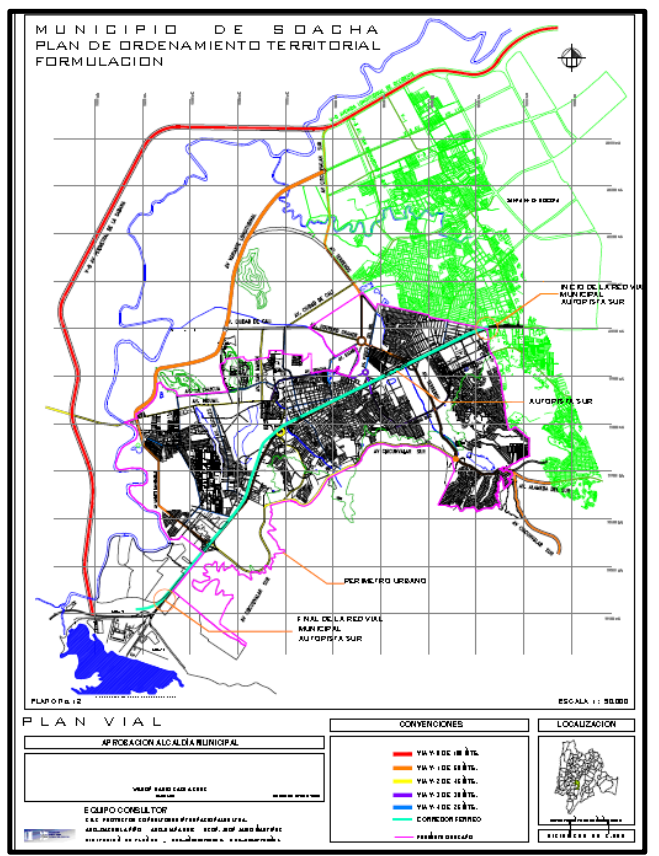

Plan Vial del municipio de Soacha

Alcaldía de Soacha, Acuerdo 46 de 2000

Soacha cuenta con un "alto déficit de vivienda social, relacionada con el crecimiento migratorio, la ubicación de asentamientos humanos en zonas de alto riesgo, zonas de ronda de las lagunas y humedales y el fenómeno de ciudad dormitorio. Adicionalmente no cuenta con equipamientos necesarios para hacer frente a la necesidad de lograr un nivel infraestructural lo suficientemente flexible y dinámico como para estar en disposición de responder a los continuos cambios que se producen a nivel socio económico" (Soacha, Acuerdo 46 de 2000), situación que desencadena escenarios de vulnerabilidad social principalmente para la población migrante que ha recibido durante la últimas décadas. 
En el artículo 23 define las zonas de amenazas y riesgos en aquellas de origen antrópico como la explosión por zona industrial y amenaza de accidentalidad por tránsito; y las de origen natural en amenaza por deslizamiento, inundaciones y rompimiento de presas de embalses. Sin embargo, dado los altos índices de densidad poblacional sumada a la proliferación de asentamientos informales, los planes de contingencia presentados para los diferentes tipos de amenaza son insuficientes para atender de manera efectiva a las potenciales víctimas de éstos fenómenos.

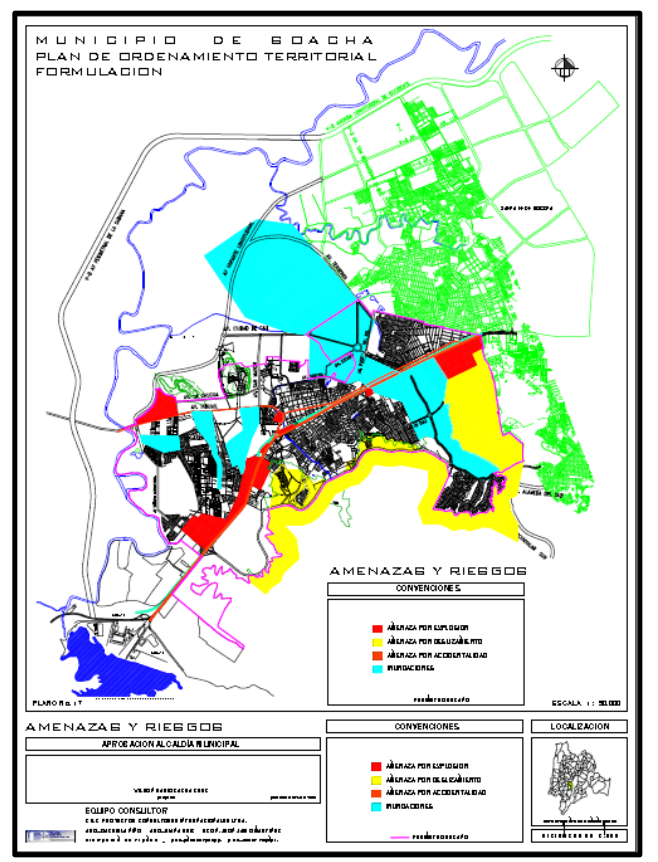

Mapa de Amenazas y Riesgos

Alcaldía de Soacha, Acuerdo 46 de 2000

\subsection{CARACTERIZACIÓN DEL MACROPROYECTO CIUDAD VERDE (SOACHA)}

Está ubicado en la Comuna 3 La Despensa en el oriente de la ciudad, Recibe su nombre debido a que alberga uno de los centros industriales más importantes del municipio y el principal puntos de conexión intermodal en términos de movilidad entre Soacha con Bogotá. Es considerada como una de las comunas más influyentes del municipio de Soacha, no solo por su proximidad a la localidad de Bosa de Bogotá y estar ubicada sobre un eje vial de nivel nacional como la Autopista Sur. Sino porque a partir de los indicadores entregados por el DANE en el Censo de 2005, la cobertura en servicios públicos domiciliarios esta entre el $88 \%$ y el $98 \%$ según el servicio; la tipo de las viviendas es del $68 \%$ casas y el $27 \%$ apartamentos; el $50,8 \%$ de la población de 3 a 5 años asiste a un establecimiento educativo formal; el 92,6\% de la población de 6 a 10 años y el 86,1\% de la población de 11 a 17 años; y el $18,7 \%$ de los establecimientos se dedican a la industria; el $50,1 \%$ a comercio; el $30,6 \%$ a servicios y el $0,6 \%$ a otra actividad, entre otros indicadores lo cual permiten inferir que su calidad de vida y actividades económicas tienen un alto nivel de desarrollo y consolidación.

El área del Macroproyecto hacia parte del suelo rural de la comuna, en la zona denominada como la Chucuita, sin embargo, desde el 2009 la clasificación del suelo se modifica para convertirse en suelo de expansión y permitir el desarrollo del Macroproyecto; lo anterior viabilizado por el artículo 3 del Decreto 4260 de 2007 del Ministerio de Ambiente, Vivienda y Desarrollo Territorial, el cual establece que los determinantes de un MISN son normas de superior jerarquía y se entenderán incorporados a los Planes de Ordenamiento Territorial de los municipios.

Así, Ciudad Verde es el segundo Macroproyecto de Vivienda de Interés Social más grande del país con 327,96 hectáreas de área bruta y el primero en potencial de soluciones de vivienda con 48.156 unidades habitacionales; se clasifica entre los proyectos de primera generación (Plan Nacional de Desarrollo 2006-2010), los cuales buscan la creación de unidades habitacionales con utilidad pública e interés social orientadas a la ejecución de una operación urbana de gran escala, con capacidad de generar impactos en el conjunto de la estructura espacial 
urbana de orientar el crecimiento general de la ciudad. Este es adoptado por medio de la Resolución 1577 de 2008 "por medio de la cual se anuncia, por motivos de utilidad pública e interés social, el Macroproyecto de Interés Social Nacional Ciudad Verde del municipio de Soacha departamento de Cundinamarca".

Para establecer su ubicación y diseño, se tuvieron en cuenta consideraciones como el crecimiento extensivo del municipio, con bajo promedio de altura y malas condiciones constructivas en casi la cuarta parte de las viviendas del municipio; poseer el mayor déficit cualitativo y cuantitativo de vivienda frente al resto del departamento de Cundinamarca y Bogotá, por lo cual urge implementar soluciones de vivienda de interés social. Adicionalmente es el municipio con mayor población de la sabana; el impacto territorial de la intervención propuesta se fundamenta en su ubicación estratégica dada su proximidad con la localidad de Bosa, la cercanía a vías reales y proyectadas de la malla vial del municipio, del Distrito Capital y de integración regional

En este sentido, el Macroproyecto Ciudad Verde buscaba promover la construcción equilibrada de vivienda, servicios urbanos y actividades productivas, mediante la generación de suelo urbanizado con condiciones de precio que garanticen el acceso a la vivienda de los hogares de más bajos ingresos; pero también surge como respuesta a la problemática social y habitacional que enfrenta el municipio de Soacha y coincide con los programas de lucha contra la pobreza extrema que han iniciado diferentes entidades públicas en escala local, regional y nacional, principalmente para la reubicación de hogares que se encuentran localizados en las zonas de alto riesgo no mitigable, atención a la población en situación de desplazamiento y mitigación parcial del déficit cuantitativo de vivienda (Biblioteca de Obras Argos, 2016).

Se ubica en el límite entre el municipio de Soacha y la localidad de Bosa en el suroccidente de Bogotá, lo que ha contribuido a consolidar el proceso de conurbación entre el Distrito y el municipio; su cercanía con Bogotá fue determinante en la viabilidad del proyecto, debido a que cuenta con preexistencias en términos de infraestructura de espacio y servicios públicos domiciliarios lo que permitiría su futura adecuación y ampliación. Está localizado en la comuna 3 del municipio de Soacha, con un área de 327,9 hectáreas de las cuales se destina el $65 \%$ del área útil al uso residencial, y gracias a ello genera un potencial de 42.000 unidades habitacionales, que podrían beneficiar a 159.180 personas aproximadamente. Este proyecto cuenta con un énfasis en vivienda social pues destina el 70\% de las unidades para VIS (29.226) y el 30\% (12.774) para VIP (Padilla, 2011: 22).

\begin{tabular}{|l|r|}
\hline AREA BRUTA & $327 \mathrm{Ha}$ \\
\hline Malla Vial Arterial & $47 \mathrm{Ha}$ \\
\hline Cuerpos Hídricos & $8 \mathrm{Ha}$ \\
\hline AREA NETA URBANIZABLE & $272 \mathrm{Ha}$ \\
\hline Parques públicos & $57 \mathrm{Ha}$ \\
\hline Equipamientos comunales & $27 \mathrm{Ha}$ \\
\hline Malla Vial Local & $29 \mathrm{Ha}$ \\
\hline AREA UTIL & $160 \mathrm{Ha}$ \\
\hline Vivienda & $107 \mathrm{Ha}$ \\
\hline Equipamientos Privados & $18 \mathrm{Ha}$ \\
\hline Comercio y Servicios & $35 \mathrm{Ha}$ \\
\hline
\end{tabular}

Áreas Generales Ciudad Verde

Espinosa, 2014: 46 - 47

Su esquema de gestión está conformado por siete etapas, que se componen de supermanzanas en las cuales se desarrollan los diferentes proyectos habitacionales, las cuales se construyen por diferentes constructoras, cada una de estas etapas contribuye a la configuración de los sistemas públicos de infraestructura aportando el suelo e incluyendo en su presupuesto la ejecución de las obras de servicio y espacio público (Padilla, 2011, 29). 


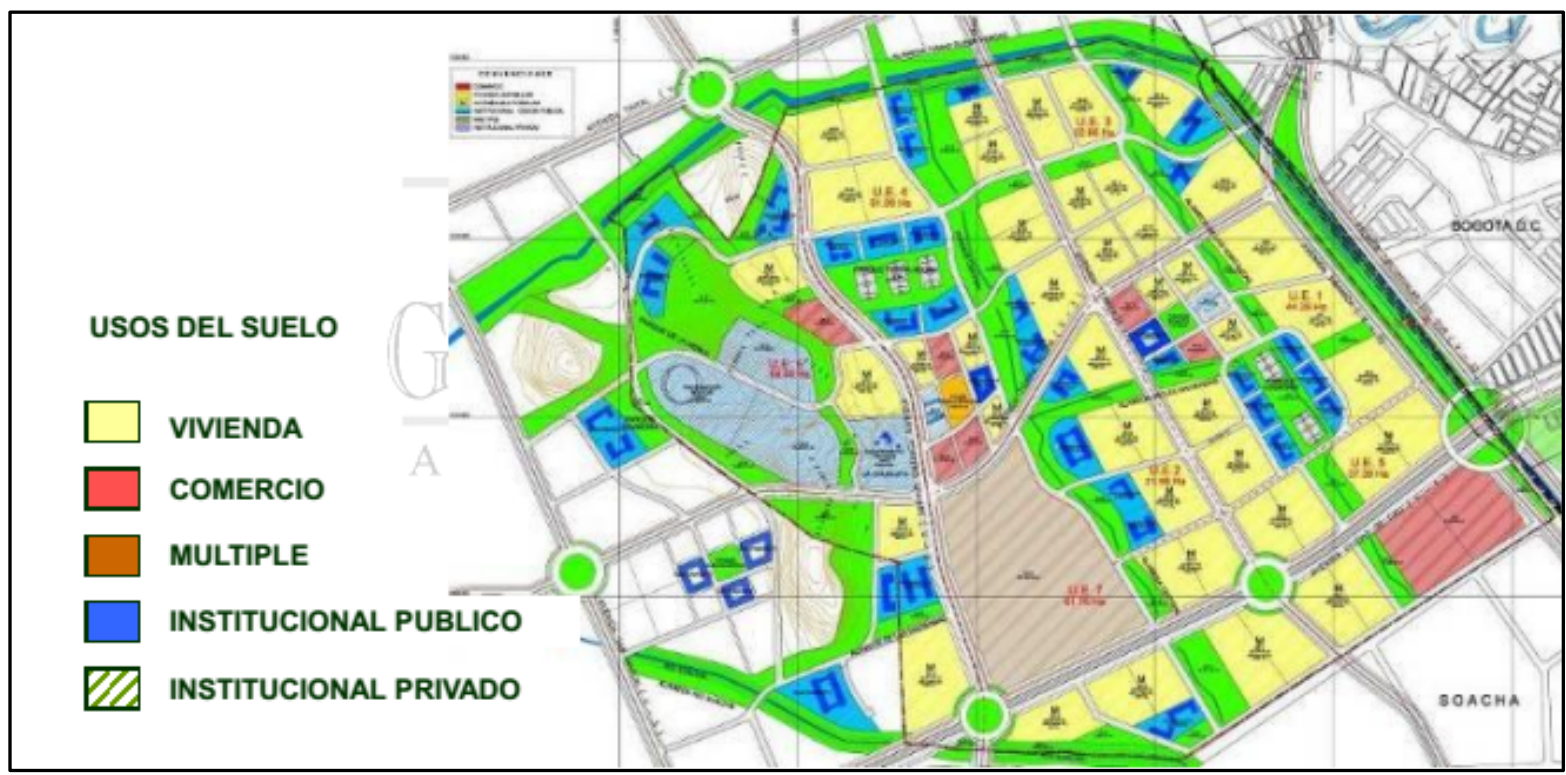

Usos del Suelo Ciudad Verde

Amarilo - Ficha técnica del proyecto Ciudad Verde, Gestión y Promoción

\section{CARACTERIZACIÓN MULTIDIMENSIONAL DEL MUNICIPIO DE MANIZALES (CALDAS)}

El surgimiento de Manizales se remonta al año 1834, a raíz de los procesos de colonización antioqueña y se funda en lo que hoy se conoce como Salamina, extendiéndose hasta el sur hasta en el cerro San Cancio. Posteriormente para 1842 la colonización se extiende hasta el sector de Morrogacho, estableciéndose familias en los que hoy son los barrios Chipre y La Francia. Ya para 1847 se comenzó la exploración del Nevado del Ruíz y en 1849 se sanciona la ordenanza para la fundación del municipio por el gobernador provincial de Antioquia (Alcaldía de Manizales, 2016).

Para principios del siglo XX, el presidente Rafael Reyes por medio de la ley del 11 de abril de 1905, escinden las provincias del Sur de Antioquia y las provincias de Robledo y Marmato del Cauca, y posteriormente crea el departamento de Caldas con capital Manizales. Hacia 1966 se divide Caldas, que sería el denominado viejo Caldas, dando origen a los departamentos de Risaralda y Quindío (Alcaldía de Manizales, 2016).

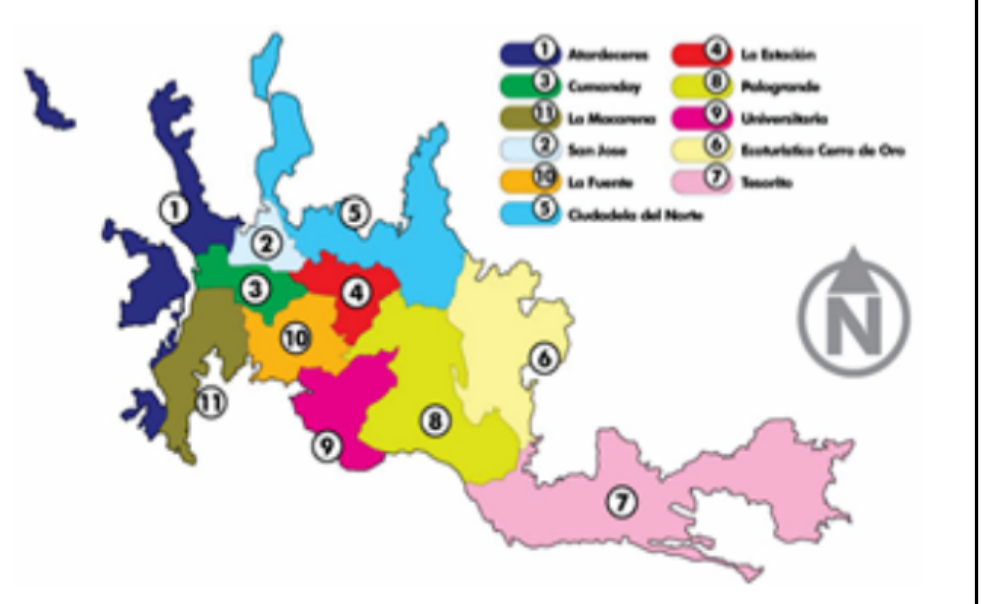

Comunas del Casco Urbano de Manizales

Alcaldía de Manzanares, 2014

Adicionalmente, el departamento de Caldas se ubica en la zona centro-occidente de la región andina de Colombia. Tiene una superficie total de $440 \mathrm{Km}^{2}$, de los cuales $60 \mathrm{Km}^{2}$ corresponden al casco urbano y $380 \mathrm{Km}^{2}$ a la zona 
rural (Duque, et al,2000). Está ubicado a una altura de $2.150 \mathrm{msnm}$. Limita al norte con el municipio de Neira, al sur con el municipio de Villa María, al occidente con los municipios de Palestina y Anserma, y al oriente con el municipio de Marulanda. El casco urbano está conformado por once comunas que albergan 114 barrios y el área rural por siete corregimientos en la zona rural; las comunas se denominan Comuna 1 (Atardeceres), Comuna 2 (San José), Comuna 3 (Cumanday), Comuna 4 (Estación), Comuna 5 (Ciudadela del Norte), Comuna 6 (Eco Turístico Cerro de Oro), Comuna 7 (Tesorito), Comuna 8 (Palogrande), Comuna 9 (Universitaria), Comuna 10 (La Fuente) y Comuna 11 (La Macarena) (Alcaldía de Manizales, 2016).

Con lo anterior, para el año 2015, según proyecciones del censo DANE 2005, la población total de Manizales era de 396.975 habitantes, de los cuales 368.633 se ubican en el casco urbano, lo que equivale al $92,85 \%$ de la población, generando una densidad poblacional de 900 habitantes por $\mathrm{km}^{2}$; el excedente se encuentra en su área rural. Considerando lo anterior, existe una estrecha relación entre las características fisiográficas del territorio y la población, que ha sido determinante para consolidar un procesos de densificación más alto.

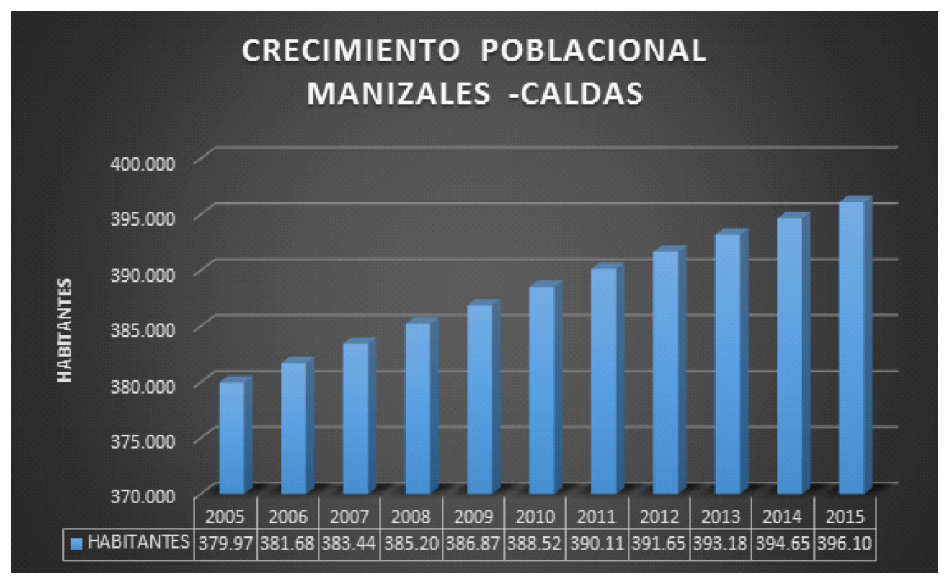

Crecimiento Poblacional

Elaboración propia a partir de datos DANE

Las áreas periféricas de la ciudad han sido el resultado de procesos migratorios de principios del siglo XX, que trajeron población en busca de los beneficios del desarrollo que estaba llevando a cabo. El casco urbano de la ciudad de Manizales se caracteriza por estar ubicado sobre un sistema montañoso que dificulta la producción de vivienda y el incremento de espacio público. Ello ha llevado a una fragmentación espacial dentro del casco urbano, generando una serie de bordes urbanos con diferenciaciones socioeconómicas. De esta forma, se configuran periferias urbanas, caracterizadas por agrupaciones de asentamientos informales, barrios de escasos recursos, Viviendas de Interés Social (VIS), condominios y asentamientos neorurales. (González-Plazas, 2009, p. 106).

Manizales, por su ubicación geográfica sobre la Cordillera Central es susceptible a varios riesgos naturales como deslizamientos, incendios, sectores in inundables (por ríos Chinchiná y Olivares), sismos, y procesos de erosión en las laderas perimetrales del casco urbano (Giraldo, 1994). De estos, los deslizamientos son los que registran el mayor número de eventos, constituyéndose en el principal riesgo que afronta la ciudad, seguido por el riesgo por inundación.

La red vial de la ciudad se encuentra dividida en vías arterias principales, vías secundarias, vías colectoras y vías locales (Concejo de Manizales, 2007, art. 33). Sobre las vías arterias principales se ha desarrollado el crecimiento de la ciudad y son las que soportan los mayores flujos urbanos especialmente las carreras que van de oriente a occidente y cuyo diseño ha respondido a las características físicas del relieve. Estas vías son fundamentalmente las carreras 14 y 23, en las que se nombran distintos tramos como la Avenida Kevin Ángel, Avenida Gilberto Alzate, Avenida Santander, Avenida Paralela,Variante Sur, Vía al Magdalena, Avenida Alberto Mendoza, Avenida Colón, Conexión Fundadores y Carrera 18; de estas se desprenden las vías secundarias que conectan los sectores de la ciudad con las vías arterias principales. Las vías colectoras por su parte, vinculan las vías arterias con las vías locales, permitiendo el acceso directo a las áreas residenciales, institucionales y recreacionales.

La población empezó a asentarse en función de las vías principales que se fueron desplegando a medida que 
aumentaba el desarrollo comercial e industrial de tipo regional. De igual forma, muchas vías fueron estableciéndose en función de los crecimientos urbanísticos que requerían mejorar la conectividad en el sentido oriente - occidente, apareciendo de nuevo asentamientos tanto formales como informales. Es decir, a medida que aumenta la población y las viviendas, no solo se requerirán soluciones viales, sino que a su vez las vías se convierten en un atractivo para edificar en cercanías a ellas por las ventajas de conectividad que ofrecen, haciendo que el fenómeno adquiera un carácter cíclico, viabilizado por el aumento de la población.

De este modo y de manera conjunta, población y sistema de movilidad, consolidan a la ciudad como eje comercial e industrial cafetero en la región; lo que a la postre ha incidido para que las dinámicas económicas impacten en términos urbanísticos a través de incrementos en el valor del suelo, cambios de uso y tratamientos urbanos e intervenciones asociadas con la renovación urbana.

\subsection{CARACTERIZACIÓN DEL MACROPROYECTO SAN JOSÉ (MANIZALES)}

La Comuna San José (antes Comuna 2) está compuesta por siete barrios: Avanzada, Asís, Delicias, Colón, Estrada, San Ignacio y San José, y durante las últimas décadas ha mostrado un serio deterioro caracterizado precisamente por la presencia de asentamientos informales en zonas de alto riesgo en su zona nororiental y por una utilización inconveniente del espacio público en su parte sur occidental.

Es precisamente a raíz de ésta situación que surge el Macroproyecto San José, el cual se desarrollará en el área ubicada en el centro tradicional de la ciudad de Manizales, abarcando la franja norte del mismo, en la cual tienen asiento desde inicios del siglo pasado los barrios San José, La Avanzada, Las Delicias, Colón, Asís, Galán, San Ignacio, San Vicente de Paúl, El Tachuelo, también hacen parte del área los barrios: Santa Helena, Fundadores y Centro, bajo la implementación de diferentes tratamientos urbanísticos, principalmente el de Renovación Urbana, el cual modifica la construcción social del hábitat y las relaciones que se han tejido con el territorio.

El Macroproyecto fue aprobado por el concejo municipal según el Acuerdo 680 de 2008, para ser ejecutado bajo el Plan de Desarrollo 2008 - 2011; como objetivo principal contempla la renovación y densificación de la zona norte del centro de la ciudad de Manizales, para generar suelo urbano, especialmente para Vivienda de Interés Social y Prioritaria, al tiempo que promoverá nuevos desarrollos integrales tendientes a mejorar las condiciones de vida de las población, a través del reasentamiento de familias localizadas en zonas de alto riesgo, el incremento del índice de zonas verdes por habitante y la dotación de equipamientos e infraestructura vial y de servicios.

Para tal fin contempla como principales estrategias la reubicación de la población asentada en las zonas de riesgo de la ladera norte y la recuperación y preservación de esta zona de valor ambiental y paisajístico; la intervención de la Avenida Colón con el propósito de generar suelo para el desarrollo de actividades económicas múltiples; impulsar el desarrollo de diferentes tipos de proyectos a fin de recuperar y crear nuevas dinámicas en el centro de la ciudad; promover la participación de los propietarios, promotores y colectivos sociales en los diferentes programas y proyectos de renovación y recuperación urbana de la zona; y además promover la incorporación de la ladera norte y de los nuevos espacios públicos desarrollados a los Sistemas Ambientales y de Espacio Público contemplados en el POT. 


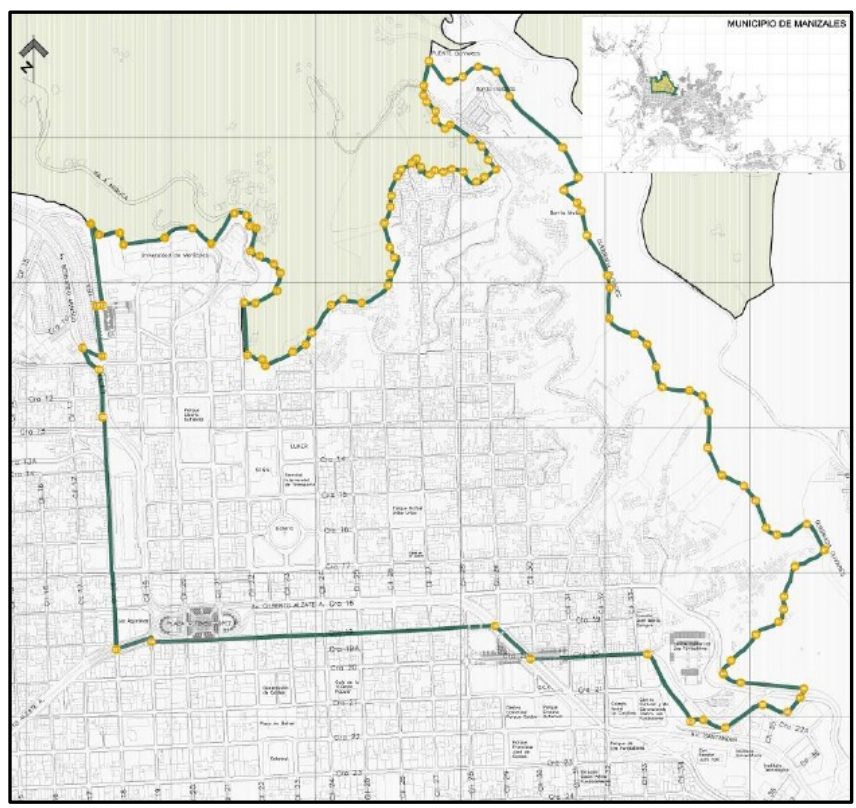

Plano de la localización y límite Macroproyecto San José

ERUM Manizales, 2016

El Macroproyecto limita al norte con la quebrada Olivares (sectores norte, nororiente y noroccidente), al sur limita con la carrera 19, al oriente limita con la calle 34 en confluencia de la Avenida Gilberto Álzate Avendaño, sector Los Fundadores, y al occidente limita con la Calle 18, Iglesia los Agustinos, Campus de la Universidad de Manizales (ERUM Manizales, 2016).

La extensión del Macroproyecto es de 110 hectáreas, "con características físico espaciales heterogéneas en tanto cuenta con edificaciones de diversa tipología por tamaño, morfología y estado de la construcción, ocupadas con usos múltiples tales como: vivienda, comercio, servicios, institucional, industrial, entre otros". Para establecer la delimitación del área, se adelantó un procesos a través de talleres entre el equipo de gobierno de la administración municipal y nacional, liderado por la Empresa municipal de Renovación Urbana ERU, el grupo de trabajo de Macroproyectos del Ministerio del Ambiente la vivienda y el Desarrollo Territorial, y el colectivo consultor de la Universidad Nacional de Colombia Sede Manizales, con lo cual se logró la materialización del ejercicio de planeamiento propuesto en el POT denominado "Piezas Intermedias de Planificación, PIP" (ERUM Manizales, 2016).

\begin{tabular}{|l|r|r|}
\hline \multicolumn{3}{|c|}{ CUADRO DE ÁREAS DEL MACROPROYECTO } \\
& \multicolumn{1}{|c|}{ M2 } & \multicolumn{1}{|c|}{ HA } \\
\hline AREA BRUTA & $1.110 .538,96$ & 111,04 \\
\hline UEU 1 - Avenida Colon y Zona Mixta & $267.751,64$ & 26,77 \\
\hline UEU 2 - Avanzada & $154.403,34$ & 15,44 \\
\hline Plan Parcial Galería & $94.138,82$ & 9,41 \\
\hline Plan de Manejo Ambiental & $230.842,48$ & 23,08 \\
\hline Pieza Intermedia de Planificación & $363.402,68$ & 36,40 \\
\hline & & MA \\
\cline { 2 - 4 } & \multicolumn{1}{|c|}{ M2 } & \multicolumn{1}{|c|}{ HA } \\
\hline Avenida Colón & $123.469,23$ & 12,35 \\
\hline Espacio publico & $33.789,12$ & 3,38 \\
\hline Equipamientos & $75.170,70$ & 7,51 \\
\hline
\end{tabular}

Cuadro de áreas del Macroproyecto San José

Alcaldía de Manizales, 2010

La ejecución se desarrollará a través de dos Unidades de Ejecución Urbanística la UEU1 Avenida Colón y UEU2 La Avanzada, en la cuales la asignación del tratamiento de renovación urbana adquiere la connotación de utilidad pública, con lo cual se viabiliza la adquisición de predios según los instrumentos contemplados en la Ley. 
Adicionalmente, las Unidades de Planificación incorporarán un Plan Parcial de Renovación Urbana para el sector de la Galería, un Plan de Manejo Ambiental para el sector Olivares, y una Pieza Intermedia de Planificación compuesta por los sectores de San José, Las Delicias, Campo Hermoso y Liborio Gutiérrez.

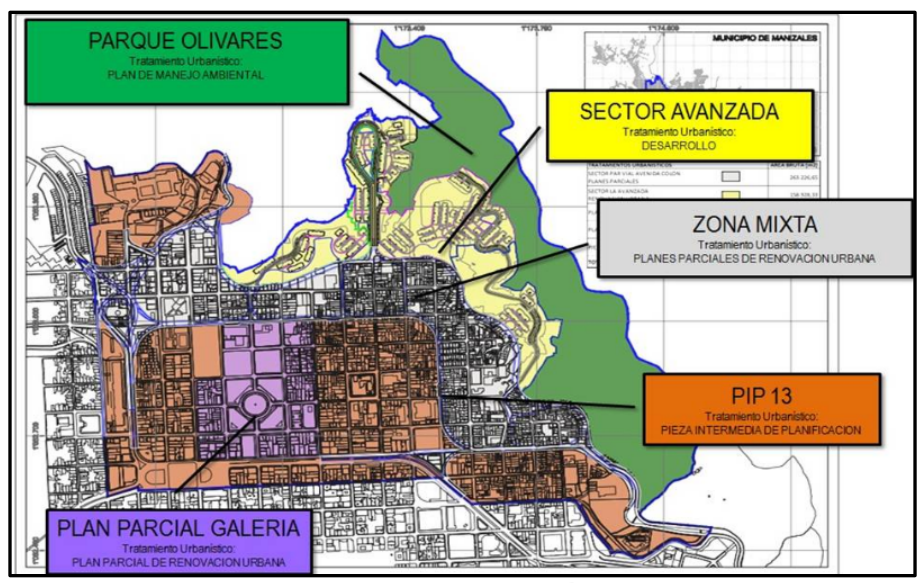

Componentes del Macroproyecto San José

ERUM Manizales, 2016

\section{IMPACTOS TERRITORIALES}

\subsection{Ciudad Verde (Soacha)}

Los primeros barrios que enfrentaron la transformación de esta zona rural en una gran macroproyecto fueron León XIII, Santa María del Rincón y Hogares del Sol; adicionalmente, con la llegada de los primeros habitantes de Ciudad Verde (aproximadamente 10.000), se empieza a evidenciar el proceso de densificación poblacional, impactando sustancialmente los sistemas de movilidad por la precariedad y deficiencias en términos de cobertura.

Por otra parte, debido al contraste generado entre un proyecto urbanístico basado en el diseño urbano y paisajístico, y los barrios de autoconstrucción o invasión colindantes, se han generado dos conflictos, en primer lugar la desvalorización de la viviendas ubicadas en barrios populares próximos a Ciudad Verde, dado que no pueden "competir" con la legalidad y planeamiento urbano que tiene el Macroproyecto; y en segundo lugar la autosegregación que genera en los habitantes de estos barrios, al comparar sus sistemas de espacio público y red vial interna. En este sentido, no solo se han evidenciado las carencias en términos urbanísticos y de provisión de bienes y servicios por parte de los habitantes de la Comuna 3 especialmente, sino que a su vez se han exigido derechos ciudadanos frente al gobierno municipal, con el fin de mitigar los procesos de segregación socioespacial al interior de la ciudad.

Adicionalmente, aunque la puesta del Macroproyecto se sustenta en un proceso de investigación técnica y socioeconómica, pone en juicio los procesos de gobernanza entre el nivel nacional y el municipal, dado que su implantación podría entenderse como una vulneración de la autonomía municipal, ante lo cual es el municipio quien no se encuentra preparado en términos institucionales para afrontar procesos como el crecimiento urbano y la densidad poblacional, sin contar con el déficit de equipamientos, baja cobertura en salud y educación, precario sistema de movilidad y aumento de asentamientos informales. Si bien dentro del reparto de cargas y beneficios se establecen cargas locales y generales a cargo de la administración y del constructor; las obras a cargo de la administración presentan retrasos por la carencia de recursos, lo que ha complicado la provisión de bienes y servicios no solo para los habitantes nativos del municipio, sino para los nuevos habitantes, afectando por ende la calidad de vida y las construcción del tejido social.

Desde el punto de vista ambiental, si bien el área no se consideraba como parte de la estructura ecológica principal del municipio, durante el proceso de construcción se retiró toda la cobertura vegetal y árboles, generando posibles afectaciones a las fuentes hídricas subterráneas por cuanto las áreas verdes contribuyen dentro del ciclo del agua. De otra parte, su cercanía a la quebrada Tibanica, hace necesaria la construcción de jarillones con el fin de evitar posibles inundaciones, lo que incorpora dos nuevo actores institucionales como la Corporación 
Autónoma Regional y la Gobernación de Cundinamarca, quienes deberán contemplar dentro del manejo de la cuenca baja del Río Bogotá, las intervenciones realizadas a sus afluentes.

En cuanto a la provisión de servicios públicos, se constituye en una de las mayores problemáticas en Soacha debido a que esta depende directamente de Bogotá, y esto se traduce en que la provisión de servicio de acueducto afecta de forma directa a ciudad verde, puesto que no llega la con suficiente presión en los últimos pisos de las torres de apartamentos lo que genera que no logre proveer del servicio a los últimos pisos de las edificaciones horizontales como las que se presentan en ciudad verde.

Este problema fue evidente desde la construcción de la primera etapa del proyecto en donde se dieron retrasos debido a que no existe conexión de servicios públicos domiciliarios haciendo que los costos de la construcción aumentaron, por la obtención de agua (en bloque) para ciertas etapas y para las demás asumir la construcción del sistema de acueducto que los conectara con la red matriz de la empresa de acueducto y alcantarillado de Bogotá y la producción de energía mediante plantas generadoras.

Durante el diseño del Macroproyecto Ciudad Verde se contemplaban las vías de acceso a este como lo son la Avenida Potrero Grande, Avenida Tierra Negra, Avenida Luis Carlos Galán, Avenida Terrero y Avenida Ciudad de Cali que permitirían el ingreso y salida de sus habitantes, especialmente hacia la ciudad de Bogotá; pero en realidad la única vía de acceso es la Avenida Terreros, que durante horas picos se satura por la cantidad de personas que se movilizan. Sumado a esto, las deficiencias en el sistema de transporte público de pasajeros, conllevan a la proliferación de sistemas de transporte ilegales que acerquen a la población hasta la Autopista Sur, afectando las economías familiares dado que implica un costo adicional o una inversión mayor en sus tiempos de desplazamiento.

Por último, aunque el Macroproyecto Ciudad Verde ha sido reconocido como uno de los proyectos bandera del gobierno nacional, por ser un ejemplo para el impulso de vivienda de interés social en el país; ha generado una ruptura con la Comuna Tres y en si con el municipio de Soacha, al no existir articulación entre la pieza urbana y su entorno inmediato, conllevando a procesos de segregación inter e intra proyecto. De manera paralela, esta ruptura se traduce en la baja accesibilidad que tienen los habitantes de la Comuna 3 a los equipamientos del Macroproyecto, así como entre los habitantes de los tipos de vivienda VIS y VIP; transmitiendo la percepción de que el proyecto diseño como una pieza aparte de la comuna y no parte de esta.

\subsection{San José (Manizales)}

Al igual que en el municipio de Soacha los primeros barrios que enfrentaron la transformación del territorio por el Macroproyecto San José, ha sido Avanzada, Galán y Asís; en donde según su estratificación socioeconómica los ubica en estratos 1 y 2 , con deficiencia en la provisión de equipamientos básicos y sociales, y una alta presencia de asentamientos informales que a través del tiempo han sido susceptibles a fenómenos de origen natural como deslizamientos e inundaciones que ubican a sus habitantes en condiciones de vulnerabilidad y restringe el uso del suelo por ser considerado de riesgo no mitigable.

Sin embargo a diferencia de Soacha, el Macroproyecto no ha alcanzado un alto porcentaje de avance debido a problemas en la estructuración financiera del mismo y a las recurrentes modificaciones de su diseño inicial, para hacerlo viable y autosostenible desde el punto de vista financiero. Situación que a la postre ha causado procesos de resistencia social y de especulación en el valor del suelo, dificultando sustancialmente la adquisición de predios para su avance.

Si bien el proyecto propone desde lo social, el mejoramiento de la calidad de vida de los habitantes de esta Comuna con el mejoramiento de viviendas y entorno urbano; desde lo ambiental, la reubicación de viviendas en zonas de alto riesgo y la recuperación del espacio público ambiental para la ciudad y desde lo urbanístico, el manejo de áreas bajo los tratamientos urbanísticos de renovación y conservación urbana, desencadenará procesos de densificación considerables y la reactivación de la zona central de la ciudad, viabilizado por el impacto que genera el par vial de la Avenida Colón sobre la pieza.

Como resultado del retraso en la adquisición de predios, se ha reconcentrado la población en el área del Macroproyecto, lo que aumenta considerablemente la vulnerabilidad frente a escenarios de riesgos de origen 
natural como deslizamientos e inundaciones, sumado a un riesgo de origen antrópico de incendio, debido a los materiales de los cuales están hechas las viviendas de esa zona, que se componen de madera, bahareque y cartón. De manera paralela, la ruptura del tejido social causada por la expectativa que generó la posible adquisición de predios por parte de la administración municipal, ha conllevado a procesos de gentrificación, pauperización de la zona y sobre todo la presencia de segundos ocupantes; dificultando más los procesos de concertación entre los habitantes y el municipio.

Por otra parte, al vincular el tratamiento de renovación urbana, gran parte de la vivienda serán reemplazadas y el entorno urbano mejorado, lo que indiscutiblemente altera lógicas de habitabilidad y de las relaciones sociales propias de la población, al pasar de una vivienda unifamiliar en un predio de gran tamaño a vivienda multifamiliares que oscilarán entre los 50 y 60 metros cuadrados y regidos bajo los parámetros de propiedad horizontal.

Adicionalmente, aunque la puesta de este Macroproyecto cuenta con un soporte técnico y socioeconómico, no solo interfiere con los procesos de gobernanza entre niveles de gobierno, sino que también ha puesto de manifiesto las falencias en la estructura de gestión y financiera del mismo y en la capacidad de la administración municipal para asumir su implementación. Lo que se ha traducido en demoras, sobrecostos, desfinanciación y críticas por parte de detractores y la comunidad académica.

\section{CONCLUSIONES}

A partir de lo establecido previamente, una de las principales causas por las cuales se han generado las ya mencionadas afectaciones en ambos macroproyectos, ha sido la falta de planeación prospectiva por parte del gobierno nacional, articulado a los niveles subnacionales; pues al "imponerse" intervenciones puntuales sobre los territorios, no solo se vulnera la autonomía municipal, sino que también se impacta la capacidad de las administraciones locales para asumir el manejo de los proyectos y la articulación de éstas piezas urbanas con el territorio y población que los habita.

Por otra parte, las preexistencias en términos de sistemas estructurantes, redes y equipamientos en la ciudad, hacen necesario que el diseño de los mismos al interior del Macroproyecto cuente con una articulación física y estrategia de financiación puntual, a fin de evitar traumatismo en la prestación de servicios básicos a los nuevos habitantes. Sumado a esto y sin desconocer las lógicas del mercado inmobiliario dominante, es necesario que desde los diferentes niveles de gobierno se diseñe estrategia para que las viviendas sean adquiridas por los propios habitantes de la zona; de este modo se conservarán los actores que conforman el tejido social de la comuna, mejorando las condiciones del hábitat y evitando desencadenar procesos de gentrificación. En este sentido, factores como el incremento en el valor del suelo resultante de las mejoras en la zona, el bajo poder adquisitivo de la población

Si bien, los dos municipios fueron sujetos de la implementación del programa de Mejoramiento Integral de Barrios bajo los lineamientos establecidos en el CONPES 3604 DE 2009, el nivel de cobertura fue muy bajo en términos de hogares y viviendas (506 para Soacha y 325 para Manizales), focalizados en un solo barrio en cada ciudad, por lo que no logra aportar información contundente sobre el impacto de este tipo de intervenciones en predios ya ocupados; por el contrario deja expectativas en torno al proceso en la población beneficiaria y circundante, pero adicionalmente como iniciativa es reemplazado por los MISN, generando procesos de resistencia social frente al tipo de operación, formas de adquisición del suelo y reconfiguración del nuevo tejido social. Dado que es una intervención que necesariamente genera densificación poblacional, conlleva por tanto a nuevas formas de reterritorialización por parte de la población original de la zona, así como de los nuevos habitantes, impidiendo la configuración de un modelo de ocupación que se articule con el contexto de cada ciudad, impidiendo se cumpla con el objetivo primario de reducir el déficit cualitativo y cuantitativo de la vivienda y el municipio.

De manera paralela las ciudades deben buscar mecanismos para mejorar la calidad de vida de sus habitantes, incentivar y atraer el desarrollo económico, bajo un acompañamiento permanente por parte del Estado; sin embargo, el diseño e implementación de políticas, programas y proyectos en materia habitacional, no necesariamente conlleva a orientar y motivar el crecimiento económico al interior de éstos municipios, puesto que no solo las inversiones en capital físico y humano estimulan el crecimiento económico, sino por el contrario deben ir asociadas a políticas específicas que fomenten la actividad productiva, regulación de mercados, y sobre todo que ofrezcan alternativas en términos de empleo y provisión de bienes y servicios a los habitantes, evitando 
convertirse en municipios dormitorio de grandes centro urbanos.

Por otro lado, la población colindante a las zonas donde se desarrollan los MISN, no tiene incidencia en la formulación y ejecución del proyecto, siendo inexistente las instancias institucionales de participación o concertación con la comunidad para el establecimiento y desarrollo del mismo, según lo establecido en la normatividad del orden nacional que da sustento a una articulación entre actores públicos y privados, sin participación de la comunidad, quienes se convierten en sujetos demandantes de unidades habitacionales

En relación con las características y alcances de los MISN, estos pretenden ir más allá que enfrentar el déficit cuantitativo de vivienda, configurándose como el mecanismo a través del cual el gobierno nacional busca reducir la informalidad y sus efectos nocivos en cuanto al acceso y disfrute de una vivienda digna; razón por la cual tanto las unidades habitacionales, como sus características y la estructura urbanística, adquieren un rol fundamental para el éxito o no de los proyectos, desconociendo las particularidades en cuanto a número de miembros o lugar de procedencia, de los hogares que las ocuparan.

Como resultante de los procesos de densificación urbana y poblacional que trae inmersos los MISN, la construcción y ampliación de la red vial, la provisión de nuevos equipamientos y la adecuación de sistemas de movilidad que poseen ciudades intermedias que han sido receptoras, genera impactos negativos en las dinámicas propias de sus territorios, por cuanto no sólo fragmenta la estructura urbana, sino que rompe el tejido social construido por sus habitantes alterando sus relaciones funcionales, al tiempo que incentiva y acelera procesos de expansión urbana con connotaciones de informalidad, bajo la expectativa de que su cercanía a un proyecto legal pueda proporcionarles el mismo carácter, como sucede principalmente en la Comuna San José de Manizales.

En este sentido, surgen interrogantes tales como ¿qué tanto se tiene en cuenta la opinión de las entidades territoriales al momento de definir un área de intervención con un Macroproyecto?, luego de su reglamentación, ¿contarán las entidades territoriales con el acompañamiento y asistencia técnica para su implementación?, dado que este tipo de intervenciones generan impactos a nivel urbano y regional, ¿están preparadas las entidades territoriales vecinas para aplicar los principios de cooperación y concurrencia frente a los procesos que desencadenan estas intervenciones?, los cuales serán resueltos a través de un proceso de seguimiento y evaluación de cada una de las etapas del Macroproyecto y la forma cómo se vincula a la ciudad para realizar la retroalimentación y ajustes del caso.

Finalmente, el déficit cuantitativo de vivienda debe suplirse en primer lugar en el municipio o ciudad objeto de la intervención y luego en las ciudades aledañas, lo anterior con el fin de evitar que se conviertan en ciudades dormitorios de otras próximas y generar relaciones pendulares que dificulten la prestación de servicios como de movilidad y provisión de equipamientos; así como la dispersión de nuevos asentamientos informales conformados por aquellos habitantes que no pueden acceder a estas soluciones de vivienda y que por el valor de eso intrínseco en las unidades habitacionales pueden llegar a incentivar escenarios de riesgo y procesos de conurbación, los que se muestran como fenómenos urbanos que han perpetuado las situaciones de miseria y dificultad de acceso a la ciudad. 


\section{BIBLIOGRAFÍA}

Acosta. P (2011) Ciudad verde los macroproyectos en acción, en Revista de Arquitectura. Número 22. págs. 2645

Acuerdo No 46 Diciembre 27 de 2000 "Por medio del cual se adopta el Plan de Ordenamiento Territorial del Municipio de Soacha”. Alcaldía Municipal de Soacha.

Alcaldía de Manizales. (2015). Proyecto de Acuerdo Por el cual se adopta la revisión ordinaria de contenidos de largo plazo del plan de ordenamiento territorial del municipio de Manizales 2015 - 2027. Componente urbano.

Alcaldía de Manizales. (2016). Sistema de Información Geográfica SIG. Recuperado de: http://sigalcaldia.azurewebsites.net/descarga_documentos.aspx.

Cantor, F. Y Cutiva, A. (2012) El plan de renovación urbana en la comuna San José en la ciudad de Manizales o el fracaso de una política pública sin público, sin ciudadanos. En X Seminario de investigación urbana y regional. Bogotá. Pontificia Universidad Javeriana.

DANE. (2010). Boletín Censo General 2005 perfil Caldas Manizales. Recuperado de: http://www.dane.gov.co/files/censo2005/PERFIL PDF CG2005/17001T7T000.PDF.

DANE. (2010). Boletín Censo General 2005 perfil Cundinamarca Soacha. Recuperado de: http://www.dane.gov.co/files/censo2005/PERFIL_PDF_CG2005/17001T7T000.PDF.

Empresa de Renovación Urbana (ERUM). 2013. Macroproyecto San José. Recuperado de: http://erum.manizales.gov.co/macroproyectosanjose.php.

Espinosa I. (2014) Los macroproyectos de interés social nacional como operaciones urbanas integrales para la política pública de vivienda. Caso ciudad Verde, área metropolitana de Bogotá. Pontificia Universidad Javeriana facultad de arquitectura y diseño maestría en planeación urbana y regional.

Fichas de Caracterización Territorial DNP (2017) disponible enhttps://www.dnp.gov.co/programas/desarrolloterritorial/Paginas/Fichas-de-Caracterizacion-Regional.aspx

Henao. M. (2011) Análisis del proceso de producción privada de vivienda de interés social (vis) en Colombia en el periodo 2007-2010 a partir del macroproyecto de interés social nacional ciudad verde. Bogotá. Colegio mayor de nuestra señora del rosario facultad de ciencia política y gobierno.

Ministerio de vivienda (2009) Resolución 1687 de 2009. Ministerio de vivienda ciudad y territorio. Colombia

Resolución 145327 de Julio (2009). Macroproyectos de interés social Nacional. Centro occidente de Colombia, San José. Bogotá. Ministerio de vivienda, ciudad y territorio.

Resolución 1527 (2016). Macroproyectos de interés social Nacional. Centro occidente de Colombia, San José. Bogotá. Ministerio de vivienda, ciudad y territorio.

Resolución 1577 (2008) utilidad pública e interés social, el Macroproyecto de Interés Social Nacional Ciudad Verde del municipio de Soacha departamento de Cundinamarca. Bogotá: Ministerio de vivienda, ambiente y desarrollo territorial. 